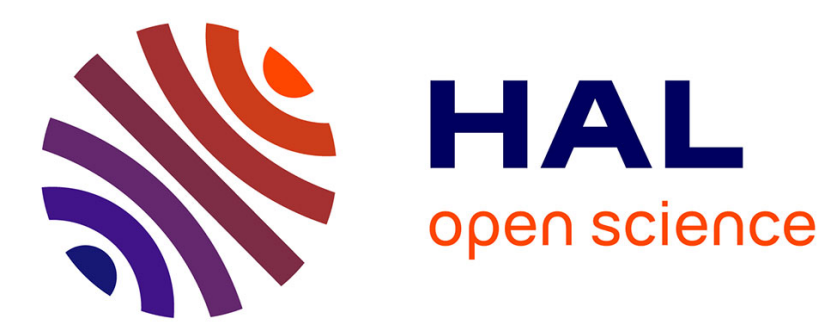

\title{
Soft X-ray reflectometry applied to the evaluation of surface roughness variation during the deposition of thin films
}

J.P. Chauvineau

\section{- To cite this version:}

J.P. Chauvineau. Soft X-ray reflectometry applied to the evaluation of surface roughness variation during the deposition of thin films. Revue de Physique Appliquée, 1988, 23 (10), pp.1645-1652. 10.1051/rphysap:0198800230100164500 . jpa-00245993

\section{HAL Id: jpa-00245993 https://hal.science/jpa-00245993}

Submitted on 1 Jan 1988

HAL is a multi-disciplinary open access archive for the deposit and dissemination of scientific research documents, whether they are published or not. The documents may come from teaching and research institutions in France or abroad, or from public or private research centers.
L'archive ouverte pluridisciplinaire HAL, est destinée au dépôt et à la diffusion de documents scientifiques de niveau recherche, publiés ou non, émanant des établissements d'enseignement et de recherche français ou étrangers, des laboratoires publics ou privés. 


\title{
Soft X-ray reflectometry applied to the evaluation of surface roughness variation during the deposition of thin films
}

\author{
J. P. Chauvineau \\ Institut d'Optique, Centre Universitaire d'Orsay, B.P. 43, 91406 Orsay Cedex, France
}

(Reçu le 19 octobre 1987, révisé le 29 janvier 1988, accepté le 2 juin 1988)

\begin{abstract}
Résumé. - Des couches minces de bore, silicium et tungstène-rhénium ont été fabriquées par évaporation sous vide. Pendant le dépôt, on a mesuré leur réflectivité en utilisant la raie $\mathrm{K} \alpha$ du carbone $(\lambda=4,47 \mathrm{~nm})$. L'évolution de la rugosité de surface a été déterminée à partir de la courbe donnant la réflectivité en fonction de l'épaisseur. On constate que l'écart quadratique moyen de la hauteur des rugosités est proportionnel à l'épaisseur des couches. Pour interpréter ces résultats, on propose un modèle statistique qui repose sur la condensation aléatoire des atomes et prend en considération le rôle de l'excès d'énergie cinétique des particules incidentes dans la croissance des couches très désordonnées.
\end{abstract}

\begin{abstract}
Thin films of boron, silicon and tungsten-rhenium materials were prepared by vapor deposition in a high vacuum system. During the deposition, the reflectivity was measured using the carbon $\mathrm{K} \alpha$ line $(\lambda=4.47 \mathrm{~nm})$. The surface roughness evolution was calculated as a function of thickness from the soft X-ray reflectance of the films. It was found that the mean square deviation of the surface height is proportional to the average thickness. A statistical model is proposed to explain this behaviour; it rests on the random condensation process of evaporated particules and takes into account the role of incident kinetic energy of adatoms in disordered thin film growth.
\end{abstract}

\section{Introduction.}

Soft X-ray reflectometry (SXR) was introduced by Spiller $[1,2]$ as a monitoring system for the deposition of multilayers for X-ray mirrors. The main advantage of SXR compared to the conventional quartz microbalance for thickness monitoring during the deposition of multilayers lies in the self-compensation of thickness errors made on the successively deposited layers. This feature of SXR is due to the fact that the amplitude of the radiation reflected by a stack of layers is the result of the interference of all the beams reflected by each of the interfaces which are reached by the incident soft X-ray beam. In the case of very thin layers as required for X-ray mirrors, the penetration depth of the incident soft $X$ ray beam can be of the order of a few tens of layers ; so, the thickness monitoring by SXR allows the production of multilayers having a large number of periods and small thickness errors.

For fixed values of wavelength and incidence angle, the reflectivity at a boundary between two layers depends on both refractive index of the materials and interface roughness. The latter is particularly important for coatings designed for short wavelength radiations. So, as pointed out by Spiller, SXR is also a good method for the in situ characterization of thin films during their deposition, especially for the surface roughness evaluation, as the wavelength of the soft X-rays used in the reflectometer is generally close to that for which the coating is designed.

Since the introduction of SXR, it has been employed for surface roughness studies in some laboratories working on soft X-ray multilayers $[2,3,4]$. In this paper we present an analysis of surface roughness variation measured during vacuum deposition of thin films of boron, silicon and tungsten-rhenium alloy selected to coat X-UV multilayer mirrors [5].

\section{Experimental.}

The film deposition is made in a high vacuum system pumped by a cryogenic pump and fitted with a double electron beam source for materials evaporation. A nearly constant evaporation rate is obtained by monitoring with a quartz crystal microbalance 
which is sensitive to the deposited mass. Films obtained from evaporated refractory materials are highly disordered; their density depends on the preparation conditions : nature and partial pressure of residual gases, deposition rate, substrate temperature. Consequently, monitoring the mass is not sufficient for an accurate thickness determination; the latter is then achieved by SXR. Thin films are deposited on flat substrates fixed on a holder which can be rotated in order to improve the thickness uniformity over the whole substrate area.

As in standard X-ray tube, soft X-rays are generated by focussing accelerated electrons with an energy of a few $\mathrm{keV}$ on a solid target. The wavelength of the emitted radiations is determined by the composition of the target material. The main lines used for deposition monitoring are $\mathrm{BK} \alpha$ $(\lambda=6.76 \mathrm{~nm}), \quad \mathrm{CK} \alpha \quad(\lambda=4.47 \mathrm{~nm}), \quad \mathrm{NK} \alpha$ $(\lambda=3.16 \mathrm{~nm})$, and $\mathrm{OK} \alpha(\lambda=2.36 \mathrm{~nm})$. The source output is about $10^{11}$ photons $\mathrm{s}^{-1} \mathrm{ster}^{-1}$ for an input power of $5 \mathrm{~W}$ and reaches

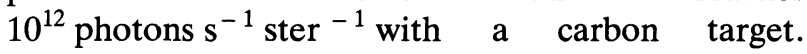
Another advantage of carbon is that the flux of emitted photons is nearly insensitive to the surface target contamination mainly due to carbonaceous compounds in usual vacuum systems. Consequently, the stabilisation of the electric power in the electron gun gives a fairly constant emission of soft X-rays as required for a subsequent analysis of the reflectivity curves recorded during the layer deposition.

Multi-anodes soft X-ray sources are commercially available. Commercial electron guns designed for material evaporation can also be used; in the second system, the wavelength is varied by changing the piece of material in the electron gun crucible. Refractory materials like carbon do not require water cooling at input electron beam power lower than about $20 \mathrm{~W}$; it is then possible to connect the accelerating high voltage to the anode and thus keep the emitting filament and associated circuitry at the ground potential. A stable soft X-ray source is then easily realized with a standard high voltage power supply and a simple amplifier controlling the grounded cathode heating power supply.

The detector is a gas flow proportional counter. After amplification and height analysis of the charge pulses generated in the detector, the peak corresponding to soft X-rays such as the $\mathrm{CK} \alpha$ line appears just above the noise level of the counting system. During the layer deposition, the high power electron gun for material evaporation can produce unwanted pulses which are eliminated by raising the lower threshold of the pulse height analyser. If the threshold level comes into the soft X-ray peak, it is necessary to avoid any drift in the peak position especially by maintaining a constant gas pressure inside the proportional counter. This can be achieved by mounting two high precision needle valves re- spectively before and after the counter in the gas line which is exhausted by a small mechanical pump.

A last point which needs some consideration in the construction of a soft X-ray reflectometer inside a deposition system fitted with an electron gun for material evaporation is that heating heavy metals like tungsten by electron bombardment produces a continuous X-ray spectrum (Bremsstrahlung). The design of the proportional counter and the choice of its position in the vacuum chamber must take into account this undesirable $\mathrm{X}$-ray source to avoid erroneous measurements.

The acceptance angle of the reflectometer, fixed by two circular diaphragms, is $0.1^{\circ}$. Then, for a grazing angle of $24^{\circ}$ the soft $\mathrm{X}$-rays scattered by surface undulations having periods lower than about $6 \mu \mathrm{m}$ are not picked up by the detector.

\section{Surface roughness measurement by SXR.}

REFLECTIVITY OF A ROUGH INTERFACE. - While the reflectivity of a plane boundary between two homogeneous media is given by the Fresnel formula, the mathematical description of the interaction of an electromagnetic wave with a rough boundary is a much more complicated problem. The measurement and calculation of the reflectivity of rough surfaces and films for grazing incidence $\mathrm{X}$-rays has been extensively studied especially by Croce et al. [6, 7]. These authors have derived rather simple analytic expressions which are valid in particular physical situations where some approximations can be used. Such a situation is encountered in the experiments reported in this paper. As for «normal » X-rays, the interaction of soft X-rays with matter is weak ; in the usual expression of the complex refractive index of materials :

$$
n=1-\delta-i \cdot k
$$

the values of $\delta$ and $\mathrm{k}$ are much smaller than 1 in the soft $\mathrm{X}$-ray range $\left(10^{-2}\right.$ to $10^{-4}$ for the $\mathrm{CK} \alpha$ line). Neglecting the multiple scattering in a rough boundary, the calculation of the reflected intensity requires only the knowledge of the statistical distribution $P(z)$ of the surface height $z(x, y)$ measured in the direction of the normal to the mean surface plane defined by $\langle z\rangle=0$. For surfaces and interfaces in films generated by random processes like vapor deposition, a good representation of the height distribution $P(z)$ is given by a Gaussian law such as :

$$
P(z)=\frac{1}{\sqrt{2 \pi \sigma^{2}}} \exp \left(-\frac{z^{2}}{2 \sigma^{2}}\right)
$$

where the variance $\sigma^{2}$ is equal to the mean square height deviation $\left\langle z^{2}\right\rangle$.

The amplitude reflectance can then be expressed by :

$$
r=r_{0} \cdot A\left\langle k_{z}, \sigma\right\rangle
$$


where $r_{0}$ is the amplitude reflectance of a perfectly flat surface and $A$ is an attenuation factor ; $k_{z}$ is the normal component of the wave vector. The factor $A$ may be explicited in the two cases where the spatial frequencies of the surface profile are respectively lower and higher than the apparent wavelength $\lambda / \sin \theta$, where $\left(\frac{\pi}{2}-\theta\right)$ is the angle of incidence at the boundary [6]. If the refraction is very weak, as for soft X-rays outside the grazing incidence range, the two different formulas of the attenuation factor have nearly the same numerical values and the reflected amplitude approximates to the common limiting expression :

$$
r=A(\sigma) \cdot r_{0}=r_{0} \cdot \exp \left\langle-\frac{8 \pi^{2} \sigma^{2} \sin ^{2} \theta}{\lambda^{2}}\right\rangle \text {. }
$$

This formula is similar to the Debye-Waller factor derived in the theory of diffraction by crystals with lattice vibrations. It is used to calculate the normal incidence relative reflectivity $R / R_{0}$ of a rough boundary as a function of the ratio $\sigma / \lambda$ (Fig. 1). The curve in figure 1 first shows the requirement of very smooth surfaces and interfaces in the optical components for soft X-ray mirrors, and moreover the high sensitivity of SXR to surface roughness variations when the mean height deviation is in the atomic scale range. For example, the intensity reflectance of a superpolished mirror having an effective surface roughness of the order of atomic dimensions $(\sigma=0.3 \mathrm{~nm})$ is reduced by half in comparison with the theoretical reflectance of a perfectly smooth boundary, when the CK $\alpha$ line $(\lambda=4.47 \mathrm{~nm})$ is used at normal incidence.

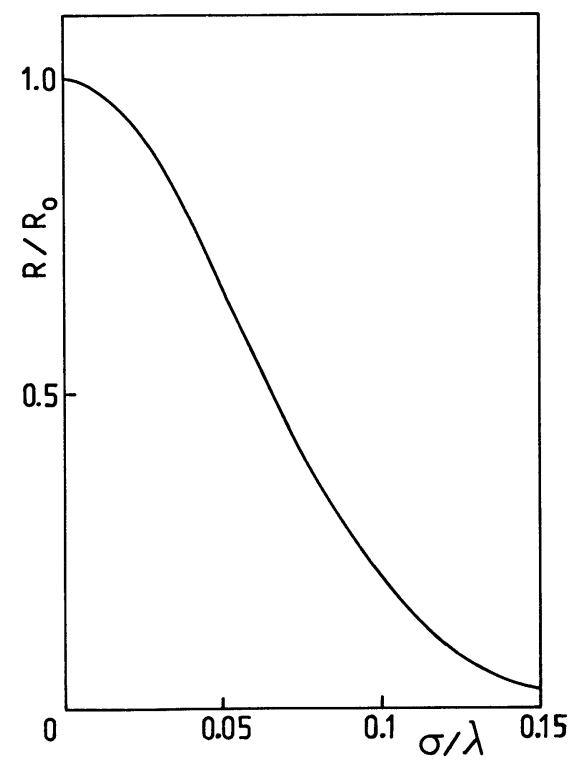

Fig. 1. - Normal incidence soft X-ray reflectance of a rough surface as a function of the standard deviation of the surface height for a normal distribution.
SOFT X-RAY REFLECTIVITY OF THIN FILMS. - The standard theory of the optical properties of thin films can be used for the calculation of the soft X-ray reflectivity of any multilayer system with perfectly flat interfaces. The general solution for the amplitude reflectance $r_{0}$ of a single film is [8]:

$$
r_{0(\mathrm{~s}, \mathrm{p})}=\frac{r_{\mathrm{t}(\mathrm{s}, \mathrm{p})}+r_{\mathrm{b}(\mathrm{s}, \mathrm{p})} \cdot \exp (-2 i \cdot \varphi)}{1+r_{\mathrm{t}(\mathrm{s}, \mathrm{p})} \cdot r_{\mathrm{b}(\mathrm{s}, \mathrm{p})} \cdot \exp (-2 i \varphi)}
$$

with $\varphi=\frac{2 \pi \cdot n \cdot d \cdot \sin \theta}{\lambda}$ where $d$ is the thickness and $n$ the complex refractive index of the film. $r_{\mathrm{t}}$ and $r_{\mathrm{b}}$ are respectively the amplitude reflectances of top and bottom boundaries of the film. If the single film is deposited on an homogeneous substrate, $r_{\mathrm{t}}$ and $r_{\mathrm{b}}$ are given by the Fresnel formulas for s-polarized and p-polarized incident light; if the substrate is a multilayer or any complex stratified medium, $r_{b}$ must be calculated step by step by a recurrent application of expression (5), starting with the Fresnel formulas from the deepest boundary in the stack.

For unpolarized incident soft X-rays, the intensity reflectance of an ideally smooth layer is :

$$
R_{0}=\frac{1}{2}\left(\left|r_{0, \mathrm{~s}}\right|^{2}+\left|r_{0, \mathrm{p}}\right|^{2}\right) \text {. }
$$

According to (5) and (6), the intensity reflected by a thin film of increasing thickness $d$ is represented by a. damped oscillating curve (Fig. 2, curve a). The pseudo-period of the oscillations approximates to $p_{0}=\frac{\lambda}{2 \sin \theta}$ as obtained by neglecting $\delta$ and $k$ in the

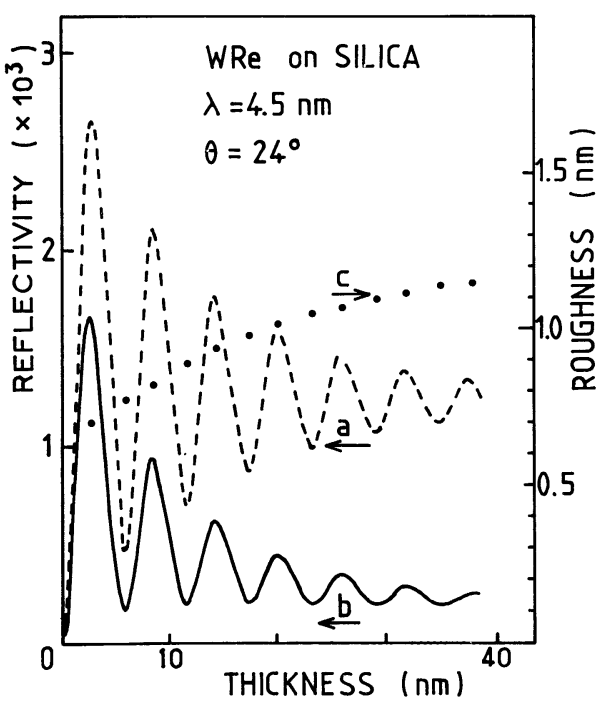

Fig. 2. - Soft X-ray reflectivity and surface roughness evolution during the deposition of WRe layer on polished silica. (a) : reflectivity calculated without surface roughness change ; (b) : experimental curve (c) : surface roughness calculated from the experimental reflectivity. 
refractive index expression (1). The amplitude decrease of the oscillations is related to the absorption of soft X-rays in the material, expressed by the imaginary part of the refractive index. The variation of the average value of the amplitude oscillations depends on the relative values of the substrate and layer material reflectivities [3].

For films having rough boundaries, the reflectance amplitude is derived from formula (5) by multiplying the Fresnel coefficients of each interface $(j)$ by the corresponding attenuation factor $A_{j}\left(\sigma_{j}\right)$ defined above. The top boundary reflectance $r_{\mathrm{t}(\mathrm{s}, \mathrm{p})}^{\prime}\left(\sigma_{\mathrm{t}}\right)$ is then readily given by a relation like (4) while the bottom boundary reflectance $r_{b(s, p)}^{\prime}\left(\sigma_{b}\right)$ must be derived step by step if the film is deposited on the top of a multilayer. As $\delta$ and $k$ are much smaller than 1 in the refractive index expression (1), we can neglect refraction and absorption for the calculation of the attenuation factor of the bottom interface from the formula (4); the factor $A_{j}\left(\sigma_{j}\right)$ is then real and depends only on $\sigma_{j}$ for fixed values of $\theta$ and $\lambda$ in the vacuum.

According to this theoretical description, the intensity reflectance $R(d)$ of real films will depend on the variation with the film thickness $d$ of the r.m.s. surface roughness amplitude $\sigma_{t}(d)$. In practice, the problem is precisely to extract informations about $\sigma_{\mathrm{t}}(d)$ from an analysis of the experimental curve representing $I(d)=I_{0} . R(d)$ where $I_{0}$ is the intensity of the incident soft X-ray beam. For example, it will be shown below that the slight decrease of the average value of the oscillating reflected intensity observed on curve $b$ in figure 2 is the result of an increase in surface roughness which occurs during the deposition of a tungsten layer on polished silica.

SURFACE ROUGHNESS OF SINGLE LAYERS ON HOMOGENEOUS SUBSTRATES. - Outside the grazing incidence range the soft $X$-ray reflectivity at a single boundary is very low. The expression giving the amplitude reflectance of a thin film deposited on a thick and homogeneous substrate is then approximately :

$$
r_{0(\mathrm{~s}, \mathrm{p})} \cong r_{\mathrm{t}(\mathrm{s}, \mathrm{p})}+r_{\mathrm{b}(\mathrm{s}, \mathrm{p})} \cdot \exp (-2 i \cdot \varphi) .
$$

This formula is derived from (5) by omitting the second term in the denominator, and so neglecting the contribution of the secondary beams due to multiple reflections in the film.

Following (4), the amplitude reflectance of real films with rough boundaries is :

$$
r_{(\mathrm{s}, \mathrm{p})} \cong A_{\mathrm{t}} \cdot r_{\mathrm{t}(\mathrm{s}, \mathrm{p})}+A_{\mathrm{b}} \cdot r_{\mathrm{b}(\mathrm{s}, \mathrm{p})} \cdot \exp (-2 i \cdot \varphi) \text {. }
$$

where the attenuation factors $A_{\mathrm{t}}$ and $A_{\mathrm{b}}$ are supposed to be independent of the soft X-ray polarization.

The intensity reflectance for unpolarized soft $\mathrm{X}$ rays is then :

$$
R_{\exp }=\frac{1}{2}\left(\left|r_{\mathrm{s}}\right|^{2}+\left|r_{\mathrm{p}}\right|^{2}\right)
$$

Following (8), the above expression (9) is a quadratic function of the unknown factor $A_{\mathrm{t}}$ which can then be calculated if all the other parameters entering in (9) are known, namely :

- the wavelength $\lambda$ and the glancing angle $\theta$ which are fixed in the experiments;

- the optical constants of the substrate and film materials needed for the calculation of $r_{t}$ and $r_{\mathrm{b}}$. They are derived from the tabulated values of the atomic scattering factors $f, f^{\prime}$ for elements in the soft $\mathrm{X}$-ray range [9], using the formula :

$n=1-\delta-i \cdot k=1-\frac{r_{0} \cdot \lambda^{2}}{2 \pi} \sum_{j} N_{j}\left(f_{j}+i \cdot f_{j}^{\prime}\right)(10)$

where $N_{j}$ is the number of atoms per unit of volume of element $j$, and $r_{0}=2.82 \times 10^{-15} \mathrm{~m}$. Besides material composition, the value of $N_{j}$ depends on the film density which can be lower than the bulk density. We have used the density values derived from the grazing incidence $\mathrm{X}$-ray characterization of the films studied in this work [4, 7]

- the intensity reflectance $\mathrm{R}_{\text {exp }}=I_{\mathrm{R}}(d) / I_{0}$ and the attenuation factor $A_{\mathrm{b}}$. These parameters are deduced from the measurement of the incident intensity $I_{0}$, the intensity $I_{\mathrm{R}}(d)$ reflected by a film of thickness $d$, and the intensity $I_{\mathrm{R}}(0)$ reflected by the bare substrate of known optical constants, as discussed above. Conversely, if the substrate surface roughnss $\sigma_{\mathrm{b}}$ is known, $A_{\mathrm{b}}$ can be calculated directly by using equation (4).

\section{Experimental results and discussion.}

With a view to realize multilayer optics for plasma diagnostics in the X-UV range, the surface roughness of thin films suitable for the fabrication of the mirror coatings was studied using SXR. Taking into account optical properties and thermal stability in multilayered structures, the selected materials are boron, silicon, and a tungsten-rhenium alloy ( $26 \%$ at. $\mathrm{Re})$. Boron was chosen mainly for its ability to give laterally graded layers of good quality for surface shape correction of mechanically polished silica substrates. Boron and tungsten-rhenium layers are deposited directly on the bare substrate while the silica surface is coated with a thin WRe underlayer before the deposition of silicon, in order to reproduce the actual conditions for WRe/Si multilayer preparation. The substrate surface roughness has been measured in a previous detailed analysis of the absolute reflectance curve recorded with the $\mathrm{CuK} \alpha$ line at glancing incidence [7]. This method was also used to determine the effective thin film density ; it was found to be from 10 to $15 \%$ lower than the bulk 


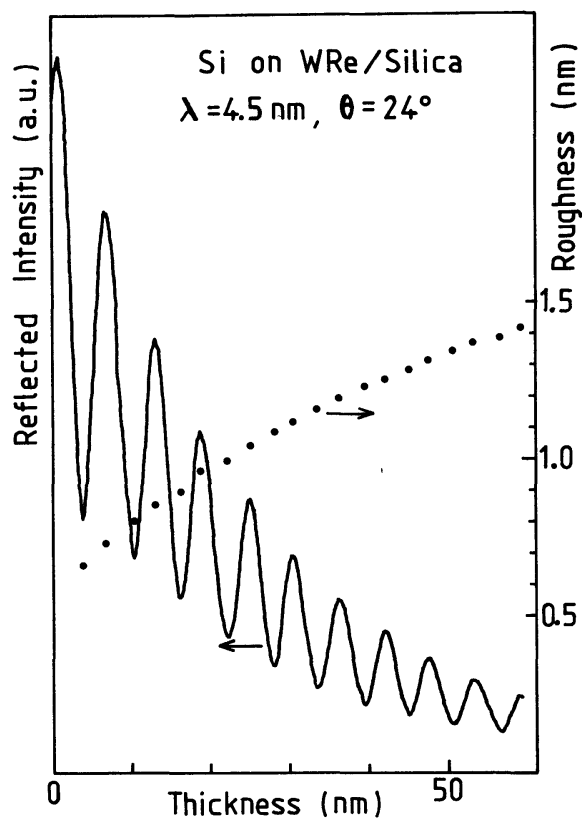

Fig. 3. - Experimental soft X-ray reflectivity (a) and surface roughness (b) changes during the deposition of silicon on polished silica coated with a thin WRe layer.

material density. The experimental values where used for the calculation of the optical constants of the films within the soft X-ray range. The reflectivity curves reported in Fig. 2.b and 3.a were obtained with the CK $\alpha$ line, at the glancing angle $\theta=24^{\circ}$. For the three materials, the surface roughness parameter $\sigma_{t}(d)$ derived from experimental reflectivity increases during the deposition of thin films (Fig. 2.c and 3.b). The rate of surface roughness development continuously decreases during the film deposition although it never reaches a saturation level at larger thickness values. A computer program using the method of least squares was applied to the determination of the analytic function $\sigma(d)$ giving the best fit for all experimental values $\sigma_{i}\left(d_{i}\right)$. The best choice is the function :

$$
\sigma(d)=\sqrt{\alpha \cdot d+\beta}
$$

where the fitting parameters $\alpha$ and $\beta$ depend on the material. This relation shows that the variance $\sigma^{2}(d)$ of the surface height $z(x, y)$ is a linear function of thickness $d$ :

$$
\sigma^{2}(d)=\alpha \cdot d+\sigma_{0}^{2} .
$$

$\mathrm{Si}$ and B thin films show a linear behaviour of $\sigma^{2}(d)$ in the whole thickness range in which reflectivity oscillations clearly appear, while for WRe the $\sigma^{2}(d)$ curve deviates from a straight line for thickness values larger than $20 \mathrm{~nm}$ (Fig. 4). The value of $\sigma_{0}$ depends on the material and is slightly higher than the substrate surface roughness $\sigma_{s}=0.6 \mathrm{~nm}$ (Tab. I).

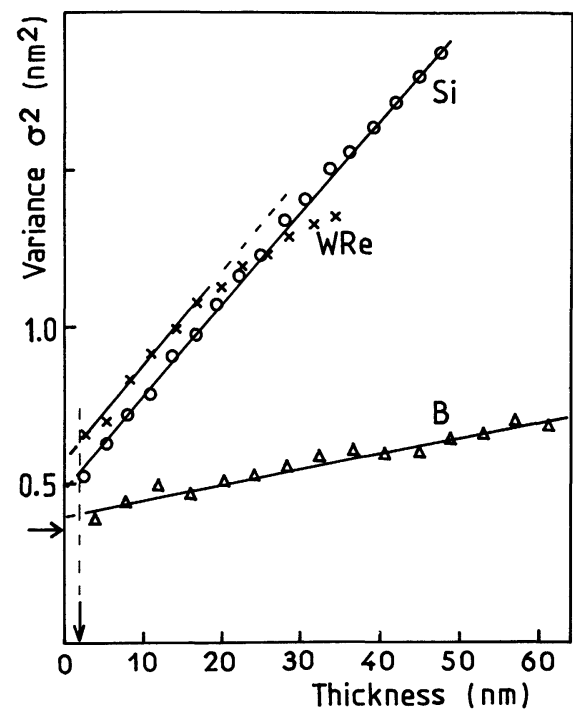

Fig. 4. - Evolution of the free surface height variance as a function of thickness during deposition of thermally evaporated boron, silicon and tungsten-rhenium thin films.

A linear variation of $\sigma^{2}(d)$ can be understood as the consequence of a random process such as atom deposition from the vapor during the film growth [13]. Let us suppose that a continuous layer is divided into a large number of vertical columns having identical square cross-section $A=L^{2}$ (Fig. 5) ; each column $(i, j)$ has a mean height $d_{i, j}=\langle z(x, y)\rangle \quad$ with $i . L \leqslant x<(i+1) . L \quad$ and $j . L \leqslant y<(j+1) . L$.

Atom condensation occurs at random intervals at the average rate $r$. During a time interval $\tau$, the average number of condensations on the area $A$ is :

$$
\left\langle N_{i, j}\right\rangle=r \cdot \tau \cdot L^{2}
$$

and the mean square deviation is :

$$
\left\langle\left(N_{i, j}-\left\langle N_{i, j}\right\rangle\right)^{2}\right\rangle=\left\langle N_{i, j}\right\rangle=r \cdot \tau \cdot L^{2}
$$

for a Poisson distribution.

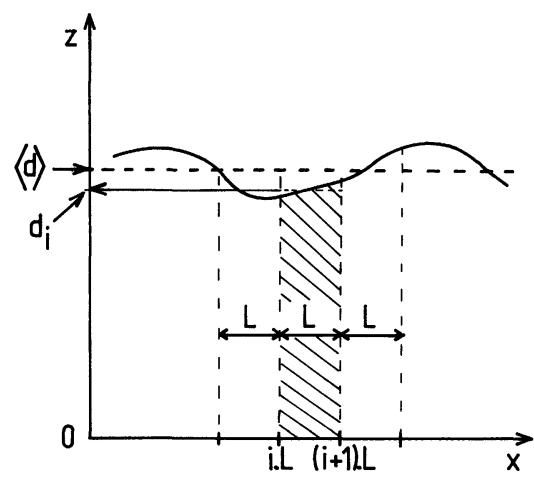

Fig. 5. - Model of a continuous rough layer deposited on a perfectly flat substrate. $\langle d\rangle$ is the mean thickness of the layer, and $d_{i}$ is the mean height of the $i$ th column. 
Let us suppose that the number of atoms in a column with cross-section $L^{2}$ is determined by the condensation process alone. The mean height $d_{i, j}$ of the column $(i, j)$ is :

$$
d_{i, j}=\frac{N_{i, j} \cdot v}{L^{2}}
$$

where $v$ is the atomic volume of the material in the layer.

It follows from (14) that :

$$
\left\langle\left(d_{i, j}-\left\langle d_{i, j}\right\rangle\right)^{2}\right\rangle=\frac{v}{L^{2}}\left\langle d_{i, j}\right\rangle=\frac{v}{L^{2}}\langle d\rangle .
$$

The variance of the column mean height is then proportional to the average thickness when the column growth is controlled by random atom condensation alone.

Let us consider first the ideal case of a continuous layer deposited on a perfectly flat substrate located at $z=0$. The film free surface height $z(x, y)$ is then identical to the local thickness $d(x, y)$. The roughness of a normally distributed surface is described by the standard height deviation $\sigma$ as defined by (2) and by the autocorrelation coefficient :

$$
C(l)=\exp \left(-\frac{l^{2}}{L_{0}^{2}}\right)
$$

where $l$ is the distance between two points $\left(x_{1}, y_{1}\right)$ and $\left(x_{2}, y_{2}\right)$ on the surface [10]. The correlation distance $L_{0}$ characterizes the lateral size of the peaks and valleys of the surface. The substitution of the real surface $z(x, y)$ for a mosaic of small squares of size $L$ and height $d_{i, j}$ will not appreciably change the statistical distribution $P(z)$ if $L$ is chosen smaller than the correlation distance $L_{0}$ for inside each column $(i, j)$ the standard deviation $\sigma_{i, j}$ of the random variable $\left(z(x, y)-d_{i, j}\right)$ will be much smaller than the overall surface height standard deviation $\sigma$ which is calculated from an area much larger than $L_{0}^{2}$. The variance $\sigma^{2}$ is then nearly equal to the column height variance expressed by (16). Consequently, the standard height deviation $\sigma$ of the intrinsic roughness of a thin film is proportional to the square root of the mean thickness if the film growth is controlled by a random process.

Considering now a rough substrate having a surface profile represented by $z_{\mathrm{s}}(x, y)$, the film surface height is now written :

$$
z(x, y)=z_{\mathrm{s}}(x, y)+d(x, y)
$$

If the substrate height distribution $P_{\mathrm{s}}\left(z_{\mathrm{s}}\right)$ is a Gaussian function with a mean height deviation $\sigma_{s}$, the variance of the sum of the two independent variables $z_{\mathrm{s}}$ and $d$ is :

$$
\sigma^{2}=\sigma_{\mathrm{s}}^{2}+\sigma_{\mathrm{f}}^{2}
$$

where the intrinsic film thickness variance $\sigma_{\mathrm{f}}^{2}$ is given by (16). The mean surface height deviation $\sigma$ is then :

$$
\sigma(d)=\sqrt{\sigma_{\mathrm{s}}^{2}+\frac{v}{L^{2}} d}
$$

where $d \equiv\langle d(x, y)\rangle$ is the mean thickness of the film.

The above expression of the surface roughness parameter $\sigma$ gives a physical meaning to the coefficients $\alpha$ and $\beta$ introduced in formula (11) derived from the experimental results. The atomic volume $v$ can be calculated from the layer density, while the correlation distance $L_{0}$ to which the length $L$ must be compared is an unknown parameter ; one can get only a rough indication about its order of magnitude by considering the physical processes liable to modify the surface shape, except for random atom condensation. There are essentially reevaporation and surface diffusion, as bulk diffusion can be ruled out given the high temperature required to change the initial structure of the thin films studied here. Reevaporation from the surface is likely to be very weak as no deposition was observed outside the evaporation field of the electron gun source. Surface diffusion can be due to an excess of kinetic energy of the incident particules, or to thermal activation once the adatoms are in thermal equilibrium with their neighbourhood. The adsorption energy increases with the number of neighbours in a vacant surface site; during a random walk on a rough surface a moving adatom has a higher probability to be fixed in the bottom of a valley than on a peak. Consequently, the surface diffusion process has a smoothing effect on a range comparable with the mean path of the moving adatoms. For incident particles, the length of the path joining the incidence point and the final fixation point is designated as the impact mobility [11].

As shown in Table I, the $L$ values calculated from the experimental parameter $\alpha$ correspond to a few interatomic distances for the three materials studied in this work. The same order of magnitude was obtained by K. H. Müller [11] for the impact mobility of Lennard-Jones particles incident on a zero temperature substrate; this theoretical ap-

Table I. - Values of parameters used in equations (11) and (20) to characterize the surface roughness increase in evaporated thin films.

\begin{tabular}{|lccccc|}
\hline Material & Substrate & $\begin{array}{c}\alpha \\
(\mathrm{nm})\end{array}$ & $\begin{array}{c}\sigma_{0}=\sqrt{\beta} \\
(\mathrm{nm})\end{array}$ & $\begin{array}{c}v \\
\left(\mathrm{~nm}^{3}\right)\end{array}$ & $\begin{array}{c}L \\
(\mathrm{~nm})\end{array}$ \\
\hline $\mathrm{B}$ & Silica & $7.2 \times 10^{-3}$ & 0.67 & $0.78 \times 10^{-2}$ & 1.04 \\
$\mathrm{Si}$ & WRe/Silica & $2.9 \times 10^{-2}$ & 0.63 & $2.0 \times 10^{-2}$ & 0.84 \\
WRe & Silica & $2.9 \times 10^{-2}$ & 0.75 & $1.9 \times 10^{-2}$ & 0.80 \\
\hline
\end{tabular}


proach uses a two-dimensional molecular dynamics simulation. As the impact mobility increases with the kinetic energy of the incident particles, it would be of interest to compare the surface roughness of similar layers made by different procedures : in vapor deposition the average kinetic energy is only a few tenth of an $\mathrm{eV}$ while it can reach several $\mathrm{eV}$ in sputter deposition. The impact mobility should also increase with the substrate temperature in accordance with the atomic model describing adatom surface self-diffusion [12]; if the atom condensation remains the only cause of surface diffusion, a substrate temperature rise during the film growth should then lead to a decrease in the slope of the $\sigma^{2}(d)$ curve. This could be a possible explanation of the shape of the curve corresponding to WRe layers (Fig 4), as the intense heat radiation emitted from the electron gun crucible causes an important rise in the substrate temperature during the evaporation of WRe.

Until now it was considered that the incident particles are impinging on continuous layers on which they are strongly adsorbed by covalent or metallic bonding for $\mathrm{Si}, \mathrm{B}$ and WRe materials. The condensation process is more complex at the beginning of the layer deposition because some atoms arrive on the bare substrate before the layer becomes continuous. The influence of the nucleation stage on the surface roughness evolution depends on the nature and density of the nucleation sites. The creation of large clusters on an initially smooth substrate will increase the surface roughness. This initial sharp rise in surface roughness was clearly observed by measuring the soft X-ray reflectivity of boron and tungsten-rhenium layers deposited on float-glass substrates [4]. Although the cluster growth stage is less important on the polished silica substrates used here than on float-glass, it could be responsible for the small difference appearing between the substrate roughness $\sigma_{s}$ and the experimentally determined parameter $\sigma_{0}$ (Tab. I and Fig. 4). The lower thickness value for which equation (20) is valid is about $1.5 \mathrm{~nm}$ for the three materials (Fig. 4). Consequently, a detailed description of the nucleation stage is essential for the explaination of the surface roughness evolution during the deposition of multilayers in which the single layer thicknesses are in the nanometer range. One can expect for example that the deposition of small nuclei in the holes of a rough substrate would reduce the surface roughness. Such smoothing effect have been observed, mainly with carbon layers $[2,3]$, but the mechanism involved is not clearly established.

More generally, the equation (20) giving the surface roughness variation during the layer deposition is not valid when the sources and sinks for the moving particules are not randomly distributed on the film surface. In crystallized thin films for example, if the temperature is sufficiently high for thermally activated surface diffusion to occur, the anisotropy of surface energy causes thermal grooving of the film free surface, which will obviously increase the surface roughness. In this case, the sources of moving atoms are localized at the emergence of defects such as grain boundaries and dislocations while the sinks are generally located on the ledges surrounding the terraces of low index crystallographic planes on the surface.

In order to compare the experimental results reported here with those previously obtained by Spiller [2] and Bruijn et al. [3] in similar experiments using SXR, we have determined the increase in surface roughness for very thin films :

$$
\Delta \sigma=\sigma(d=10 \mathrm{~nm})-\sigma(d=0) .
$$

The values reported in Table II are more or less scattered, even for the same material and substrate. As stated above, thin film deposition can include a first stage of discontinuous layer growth which is very sensitive to the structure, temperature and chemical nature of the substrate surface and influences significantly the surface roughness evolution. The comparison between results obtained on the same materials but in different experiments is then rather tricky in the absence of detailed informations concerning the substrate surface state prior to layer deposition.

Table II. - Surface roughness increase $(\Delta \sigma)$ in thin films of thickness $d$ made by vapor deposition on room temperature substrates. (Ref. $\left[{ }^{*}\right]$ : this work).

\begin{tabular}{|ccccc|}
\hline Material & Substrate & $\begin{array}{c}\Delta \sigma \\
(\mathrm{nm})\end{array}$ & $\begin{array}{c}d \\
(\mathrm{~nm})\end{array}$ & Ref. \\
\hline $\mathrm{B}$ & $\mathrm{WRe}$ & 0.1 & 20 & {$[2]$} \\
$\mathrm{B}$ & $\mathrm{Silica}$ & 0.05 & 10 & {$\left[{ }^{*}\right]$} \\
$\mathrm{Si}$ & $\mathrm{WRe} / \mathrm{C}$ & 0.6 & 10 & {$[2]$} \\
$\mathrm{Si}$ & $\mathrm{Co}$ & 0.3 & 17.5 & {$[2]$} \\
$\mathrm{Si}$ & $\mathrm{WRe} / \mathrm{Silica}$ & 0.2 & 10 & {$\left[{ }^{*}\right]$} \\
$\mathrm{WRe}$ & $\mathrm{Si}$ & 0 to 0.25 & 10 & {$[2]$} \\
$\mathrm{WRe}$ & Float-glass & -0.3 to 0.2 & 10 & {$[2]$} \\
$\mathrm{WRe}$ & $\mathrm{Si}$ & 0.7 & 35 & {$[3]$} \\
$\mathrm{WRe}$ & Silica & 0.17 & 10 & {$[*]$} \\
\hline
\end{tabular}

In conclusion, it is shown that the surface roughness increases as the square root of thickness during the deposition of $\mathrm{B}, \mathrm{Si}$ and WRe continuous thin films. This behaviour is interpreted with a model which takes into account random condensation and impact mobility of adatoms on rough surfaces. It can be expected that increasing the impact mobility 
would reduce the development of surface roughness during the deposition of highly disordered continuous thin films.

\section{Acknowledgements.}

The author whishes to thank L. Valiergue for careful sample preparation.

\section{References}

[1] Spiller, E., Segmüller, A., Rife, J., Haelbich, R. P., Appl. Phys. Lett. 37 (1980) 1048.

[2] SPIller, E., Applications of thin film multilayered structures to figured X-ray optics; G. F. Marshall Ed., Proc. SPIE 563 (1985) 367.

[3] Bruijn, M. P., Verhoeven, J., VAN DeR Wiel, M. J., Nucl. Inst. Meth. Phys. Res. 219 (1984) 603 ;

Bruijn, M. P., Muller, H., Verhoeven, J., VAN DER WIEL M. J., Surface Sci. 154 (1985) 601.

[4] Chauvineau, J. P., Corno, J., Decanini, D., NÉvot, L., PARDo, B., Applications of thin film multilayered structures to figured X-ray optics ; G. F. Marshall Ed., Proc. SPIE 563 (1985) 245.

[5] Chauvineau, J. P., Decanini, D., Mullot, M., Valiergue, L., Delaboudinière, J. P., Applications of thin film multilayered structures to figured X-ray optics ; G. F. Marshall Ed., Proc. SPIE 563 (1985) 275.
[6] Croce, P., Acta Electr. 24 (1981-82) 247 ; J. Opt. (Paris) 10 (1979) 37.

[7] Névot, L., Croce, P., Rev. Phys. Appl. 15 (1980) 761.

[8] Berning, P. H., Theory and calculation of optical thin films, in Physics of thin films, G. Hass Ed. 1 (1963) 69.

[9] Henke, B. L., Lee, P., Tanaka, T. J., Shimabukuro, R. L., Fujikawa, B. K., Atomic data and nuclear data tables 27 No. 1 (1982).

[10] Beckmann, P., Spizzichino, A., The scattering of electromagnetic waves from rough surfaces. (The Macmillan Company, New York) 1963, p. 80.

[11] MÜller, K. H., Surface Sci. 184 (1987) L375.

[12] Blakely, J. M., Prog. Mater. Sci. 10 (1963) 395.

[13] ChOPRA, K. L., Thin Film Phenomena (McGrawHill Book Company, New York) 1969, p. 185. 